\title{
Pacemaker induced post cardiac injury syndrome
}

\begin{abstract}
Post pacemaker insertion pericarditis is a rare form of post cardiac injury syndrome. It is an inflammatory process involving the pericardium and/or pleura due to a recent cardiac injury. Post cardiac injury syndrome may develop after myocardial infarction (Dressler syndrome), cardiac surgery or trauma. However, in rare cases it can occur following minor cardiac procedures. We report a case of a 74-year-old male with post-cardiac injury syndrome 4 weeks post permanent pacemaker implantation. A high index of suspicion in addition to ordering appropriate imaging studies and device interrogation are crucial to establish correct diagnosis and rule out lead perforation.
\end{abstract}

Keywords: pericarditis, pacemaker, cardiac, injury
Volume 2 Issue 4 - 2015

\author{
Mohsin Salih,' Mohamed Ayan, ${ }^{2}$ Mohamed \\ Gashouta,' Aiman Smer, ${ }^{2}$ Anthony Pearson' \\ 'Department of Internal Medicine, St Luke's Hospital, USA \\ ${ }^{2}$ Department of Internal Medicine, Creighton University medical \\ center, USA
}

\begin{abstract}
Correspondence: Mohamed Ayan, Department of Internal Medicine, Creighton University medical center, I 1017 Lafayette plaza, \# 2012, Omaha, NE 68I54, USA, Tel 832-88I-3047, Email mohamedayan@creighton.edu
\end{abstract}

Received: May 20, 2015 | Published: June 26, 2015
Abbreviations: PCIS, post cardiac injury syndrome; AV, atrioventricular; TTE, transthoracic echocardiogram; WBC, white blood cells

\section{Introduction}

Post cardiac injury syndrome (PCIS) is a well-recognized clinical entity. ${ }^{1}$ PCIS is an inflammatory pericarditis that develops a few weeks after a cardiac injury. It usually presents following myocardial infarction (Dressler syndrome), cardiac surgery or trauma. In rare cases it can occur after minor cardiac procedures. The mechanism of PCIS is still not well understood. However, an immune mediated inflammatory response to initial cardiac injury may play a role in the pathogenesis of this syndrome. ${ }^{2}$ We report a rare case of PCIS after permanent pacemaker implantation.

\section{Case presentation}

A 74-year-old male presented with symptoms of pre-syncope, shortness of breath and fatigue. His past medical history was significant for known right bundle branch block, gastroesophageal reflux disease, allergic rhinitis and chronic constipation. A 48 Holter monitor revealed type 2 second degree atrioventricular (AV) block.

On 01/14/2015, he underwent placement of a Medtronic dual chamber permanent pacemaker without any apparent complications. Postoperative device interrogation and chest $\mathrm{x}$-ray were normal; $\mathrm{Hb}$ was $13.5 \mathrm{~g} / \mathrm{dl}$. On 01/30/2015 he presented with sudden onset of racing heart and presented to the ER with atrial fibrillation with rapid ventricular response, he converted to sinus rhythm with intravenous Diltiazem. Transthoracic Echocardiogram (TTE) showed small pericardial effusion but was otherwise normal Four weeks later on $02 / 12 / 2015$, he presented with right sided pleuritic chest pain and shortness of breath. He also reported occasional palpitation but no fever, chills, cough, syncope, leg swelling or joints pain. His vital signs were stable. Physical examination was remarkable for a soft pericardial friction rub and diminished breath sounds at the lung bases. Laboratory data showed mild leukocytosis (WBC: 12.8k/ul), CRP: $12.9 \mathrm{mg} / \mathrm{dl}($ normal $<1 \mathrm{mg} / \mathrm{dl})$ and ESR: $64 \mathrm{~mm} / \mathrm{h}$. A 12 -lead ECG showed sinus rhythm with atrial tracking ventricular pacing and right bundle branch bock pattern in V1 (Figure 1). A chest x-ray revealed bilateral pleural effusion and confirmed that both leads were in appropriate positions (Figure 2). TTE revealed a moderate sized pericardial effusion with no echocardiographic finding of tamponade physiology (Figure 3).

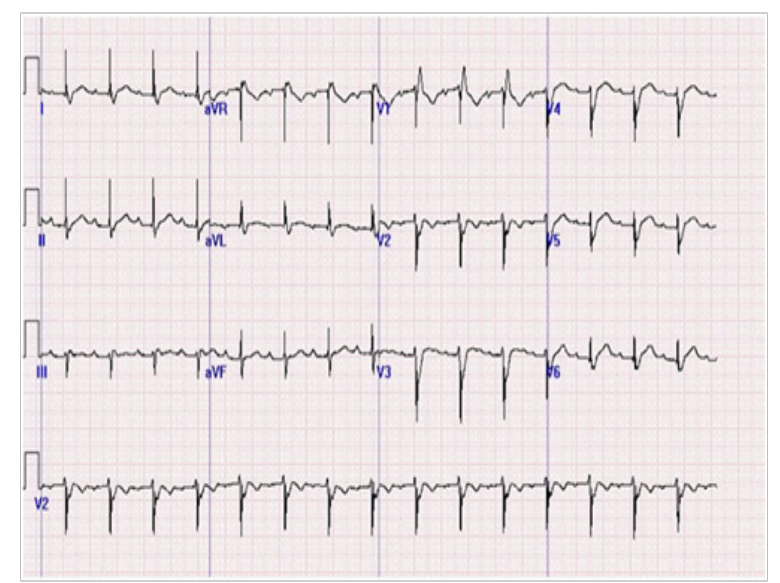

Figure I A I2-lead ECG showed sinus rhythm with atrial tracking ventricular pacing and right bundle branch bock pattern in VI.

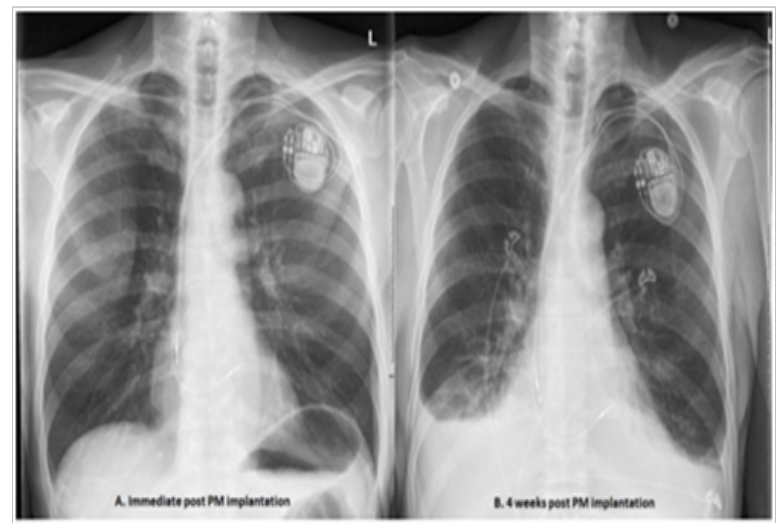

Figure 2 A chest $x$-ray showed bilateral pleural effusion and confirmed that both leads were in appropriate positions.

The patient was readmitted to the hospital. Pacemakers check demonstrated $9.7 \%$ atrial fibrillation and normal sensing and captures thresholds and lead impendence parameters. Patient underwent right sided thoracentesis with removal of 1 liter of exudative 
straw-colored pleural fluid. Cytology, gram stain and cultures were negative. He was started on colchicine with presumptive diagnosis of pleuropericarditis and his symptoms resolved shortly thereafter. The patient was discharged home in stable clinical condition. A follow up echocardiogram showed almost complete resolution of the pericardial effusion.

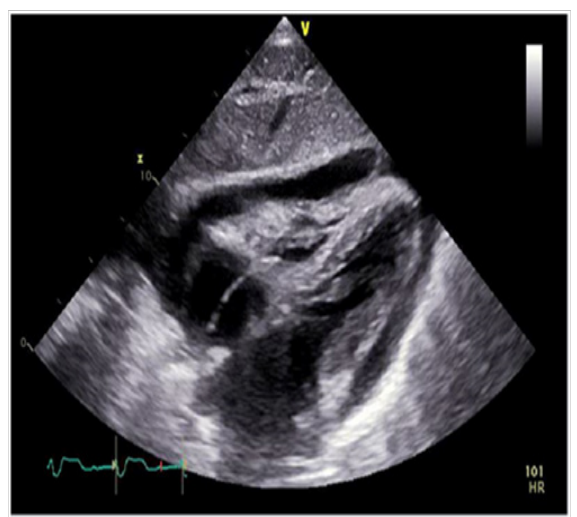

Figure 3 Transthoracic echocardiogram showed a moderate sized pericardial effusion.

\section{Discussion}

PCIS is an inflammatory process involving the pericardium and/or pleura due to a recent cardiac injury. Dressler's syndrome following myocardial infarction is the prototype of PCIS. However, it can also occur after cardiac surgery (post-pericardiotomy syndrome), cardiac trauma (post-traumatic pericarditis) or rarely post pacemaker implantation (post-pacemaker insertion pericarditis). In 1975, Donald Kaye et al described the first case of post pacemaker implantation pericarditis. ${ }^{3}$ Since then only 18 cases have been reported. Post-pacemaker insertion pericarditis is a rare form of PCIS with an estimated incidence of 1 to $2 \%$ after pacer implantation. ${ }^{4}$ The mechanism of PCIS in general and post pacemaker insertion pericarditis in particular is still not well understood.

However, an immune mediated inflammatory response to initial cardiac injury triggered by pacemaker lead may play a role on the pathogenesis of this syndrome. ${ }^{2}$ Advanced age, female gender and the use of active fixation leads are independent risk factors for the development of post pacemaker insertion pericarditis. ${ }^{5}$ The clinical manifestations are characterized by pleuritic chest pain, fever and elevated acute phase reactants, pericardial effusion and/or pleuritis with or without pleural effusion. Most of these patients present within one month after the index procedure. Physical examination may reveal distant heart sounds, pericardial or pleural rub and signs of pleural effusion. Chest $\mathrm{x}$ ray and echocardiogram confirms the presence of pleural and/or pericardial effusion as well as leads position. Overt or micro lead perforation is an important differential diagnosis and a well-known complication of pacemaker implantation. ${ }^{6}$

There are no definite criteria to differentiate between pacemaker lead perforation and PCIS without perforation. However, increased capture threshold, drop in $\mathrm{R}$ wave amplitude and marked increase or decrease in lead impedance suggest lead perforation. Nonetheless, normal pacemaker function does not rule out lead perforation. In rare occasions, lead perforation maybe visible on imaging studies. ${ }^{4}$ Early diagnosis of pacemaker induced PCIS is very important as most of these patients respond well to medical treatment with NSAID, colchicine and/or steroids. ${ }^{7}$ Although PCIS typically has a benign course, it may result in significant morbidity and mortality due to cardiac tamponade, arrhythmias and pulmonary edema.

\section{Conclusion}

Post pacemaker insertion pericarditis is a rare form of PCIS. It usually presents within one month from pacemaker implantation with symptoms and signs of pericarditis. Lead perforation is an important differential diagnosis needs to be ruled out. Although PCIS responds well to anti-inflammatory agents and has favorable prognosis, delayed diagnosis may result in potential serious complications such as cardiac tamponade. Therefore, its early detection is of clinical importance. High index of suspicion in addition to ordering appropriate imaging studies and device interrogation are crucial to establish correct diagnosis and rule out lead perforation.

\section{Acknowledgements}

None.

\section{Conflict of interest}

The author declares no conflict of interest.

\section{References}

1. Imazio M, Hoit BD. Post-cardiac injury syndromes. An emerging cause of pericardial diseases. Int J Cardiol. 2013;168(2):648-652.

2. Cevik C, Wilborn T, Corona R, et al. Post-cardiac injury syndrome following transvenous pacemaker insertion: A case report and review of the literature. Heart Lung Circ. 2009;18(6):379-383.

3. Kaye D, Frank1 W, Arditi LI. Probable postcardiotomy syndrome following implantation of a transvenous pacemaker: report of the first case. $\mathrm{Am}$ Heart J. 1975;90(5):627-630.

4. Wolk B, Dandes E, Martinez F, et al. Postcardiac injury syndrome following transvenous pacer or defibrillator insertion: CT imaging and review of the literature. Curr Probl Diagn Radiol. 2013;42(4):141-148.

5. Ohlow MA, Lauer B, Brunelli M, et al. Incidence and predictors of pericardial effusion after permanent heart rhythm device implantation:Prospective evaluation of 968 consecutive patients. Circ J. 2013;77(4):975981.

6. Banaszewski M, Stepinska J. Right heart perforation by pacemaker leads. Arch Med Sci. 2012;8(1):11-13.

7. Sedaghat-Hamedani F, Zitron E, Kayvanpour E, et al. Post cardiac injury syndrome after initially uncomplicated CRT-D implantation: A case report and a systematic review. Clin Res Cardiol. 2014;103(10):781-789. 